\title{
Audit of handover documentation during patient transfers between learning disability psychiatry community teams
}

\author{
AIMS AND METHOD \\ The local experience of having to take \\ over the care of patients newly \\ moved to the Bristol area revealed \\ the lack of clear information on their \\ complex needs. Official guidelines do \\ not exist, therefore a consensus list \\ of expected handover information \\ was produced. The records of two \\ patients were analysed in each of the \\ six teams that agreed to be audited. A \\ standardised handover form was \\ devised and introduced (Part 1). Part \\ 2 of the audit was performed 18
}

months after the implementation of changes to practice.

RESULTS
Part 1 revealed that diagnosis (11/12)
and medications ( $12 / 12)$ were the
best-documented items during
handover correspondence. Follow-up
duration $(3 / 12)$, crisis plan $(3 / 12)$,
professionals involved $(0 / 12)$ and risk
assessment $(3 / 12)$ if documented
were done so only for patients with
complex needs. Part 2 showed
improved documentation of

information ( $11 / 11$ for the first two items and 10/11 for the remainder)
CLINICAL IMPLICATIONS
Absent or concealed information in patient notes may contribute to disruption to the continuity of care following patient transfers. The use of a succinct, structured and easily distinguishable handover form in patient records may facilitate communication between professionals.

The importance of safe handover of patient information has been heavily emphasised in the care of acutely ill hospital patients. ${ }^{1,2}$ With advances in community care and the majority of psychiatric care being provided in the community, this becomes a concern in mental health services, particularly during the move of the patient from one geographical area to another. Although joint meetings where professionals hold structured and comprehensive handover sessions face to face are reassuring, it is still questionable whether the documentation of the handover information prior to care plan meetings is adequate. Some professionals propose the use of electronic patient records as a remedy for such information loss; ${ }^{3}$ however, so far in the UK, patient handover documentation for care in the community has not been standardised. Previous studies and reviews carried out in nursing settings and out-of-hours community care suggest that the use of standardised sheets and protocols reduces the risk of lost data and improves the quality of information handed over. ${ }^{4-6}$

During multidisciplinary referral meetings of our team, it was observed that important information and documentation was missing in medical notes for patients transferred from distant areas of the county. Upon discussion with medical colleagues from the Avon and Somerset area learning disability psychiatry teams, an agreement was reached regarding an audit of our own practices of handover documentation during patient transfers in the Bristol area.

\section{Method}

There was no official handover documentation guidelines at the start of the study, although there were some local verbal agreements. Therefore, in discussion with the educational supervisor and audit lead, a list of expected handover information was produced.

A sample of 12 patient records was studied. In order to ensure randomisation, the two most recently transferred patients' files prior to the audit decision were obtained from each of the six participating teams (one team did not wish to participate). This also ensured that the information audited was produced by clinicians who consented to the audit and avoided any involvement of clinicians who previously worked in the teams. Documentation regarding the following items was examined:

- diagnosis

- medications

- risk assessment

- crisis plan

- follow-up duration by the transferring team

- name and contact details of the professionals involved in the patient's care in the transferring team.

The type of official handover document was also noted (e.g. general practitioner letter, consultant-toconsultant letter).

Following Part 1 of the audit, a standardised handover form was designed with the aim of helping clinicians to transfer patient information in a succinct, structured format, distinguishable in colour from the rest of the records and therefore easily retrievable from the patient's notes. The results were presented at an academic meeting, at which a proposal was made to implement the newly devised handover form. The form was presented to the faculty members and feedback was requested. There was a mixed reception from the faculty members, with some clinicians expressing an interest in the implementation of the form, whereas others felt that its routine 
implementation would add to administrative workload and the form should therefore be used at the clinicians' discretion.

Part 2 of the audit cycle was completed 18 months after the introduction of the form, by repeating the audit process for the three teams that had implemented the form routinely. The handover documentation of these three teams was examined using the same method except that four patient records per team were requested for analysis to match the original sample number.

\section{Results}

\section{Part 1}

Diagnosis and medications were the best-documented items in the handover correspondence (Table 1). Followup duration, crisis plan, professionals involved and risk assessment were documented only for patients with complex needs. It was noted that for some transfers this information was present in parts of the medical records other than the handover documentation, but it was not easily visible or accessible and it was time-consuming to retrieve it from the abundance of clinical information present in voluminous notes. It was also noted that the type of handover documentation was varied: documentation used included copies of brief or comprehensive general practitioner letters, consultant-to-consultant letters and forensic nurse notification to the receiving team.

\section{Part 2}

Following the presentation of the results of the first audit and the newly designed form to the faculty members, three out of the initial seven teams implemented the form routinely for every patient, while in the four remaining teams the use of the form was at the clinician's discretion and was determined generally by case complexity. Eleven patient records were obtained for reaudit; the records of one patient could not be reached by the deadline owing to inability to identify the most recent transfer during long-term administrative staff leave.

The results are shown in the right-hand column of Table 1. The handover form was the main mode of information transfer and was accompanied by a brief letter for patients on a standard care plan approach (CPA) and by a comprehensive letter for patients on an enhanced CPA. There was a marked improvement in the recording of items expected to be present in the handover documentation (Table 1). Diagnosis and treatment were documented clearly in all forms; other parameters were documented in all but one form. A review of the use of the form during this process led to minor amendments to increase its practicality.

\section{Discussion}

These audit procedures aimed at taking a 'snapshot' of one aspect of the service, handover of information, by
Table 1. Patients' records containing expected information in handover documentation

\begin{tabular}{|lcc} 
& \multicolumn{2}{c}{$\begin{array}{c}\text { Patient records containing } \\
\text { the expected information, } n\end{array}$} \\
\cline { 2 - 3 } Information expected & $\begin{array}{c}\text { Part 1 } \\
(n=12)\end{array}$ & $\begin{array}{c}\text { Part 2 } \\
(n=11)\end{array}$ \\
\hline Diagnosis & 11 & 11 \\
Medications & 12 & 11 \\
Risk assessment & 3 & 10 \\
Crisis plan & 3 & 10 \\
Follow-up duration & 3 & 10 \\
Professionals involved & 0 & 10 \\
\hline
\end{tabular}

one senior house officer during 6-monthly training posts. Because of this specific target, as well as time and personnel constraints, the number of randomly selected patient records was limited to 12 . The audit showed that documentation of essential information in an easily accessible format is important during patient transfers between community teams. During Part 1 of the audit cycle it also became clear that some of the necessary information was present in patient notes but was concealed in the voluminous patient records. It was time-consuming to find and retrieve this information for receiving clinicians. The results following the implementation of a structured form indicate that disruption to flow of information and lack of clarity about the patient's background during inter-team transfers can be partly eased by the use of structured, succinct, standardised forms which are also in a format easily distinguishable (e.g. by colour) from the rest of the patient records.

Only three of the original six teams took part in Part 2 of the audit cycle. The use of a structured form was perceived by the remaining teams as extra administrative work which could be avoided by improving letter-writing. However, all these teams acknowledged the shortfalls highlighted by Part 1 and pledged to address these shortfalls by giving consideration to audit results while writing their traditional handover letters. To address these concerns, the form can be used to replace any further correspondence and the use of accompanying letters can be at the clinicians' discretion.

\section{Declaration of interest}

None.

\section{References}

1 Weinstock M. Save lives now. Patient care. Transitioning care Hosp Health Netw 2005; 79 : 27-8.

2 Solet DJ, Norvell JM, Rutan GH, Frankel RM. Lost in translation: challenges and opportunities in physician-to-physician communication during patient 
handoffs. Acad Med 2005; 80 : 1094-9.

3 Mooney M, Barry S, Draper R. The electronic patient record as a guarantor of personalised menta health care. Medinfo 1998; 9 . $14-7$.
4 Davies S, Priestly M. A reflective evaluation of patient handover practices. Nurs Stand 2006; 20 $49-52$.

5 King N, Thomas K, Bell D. An out-of-hours protocol for community palliative care, practitioners' perspectives. Int J Palliat Nurs 2003; 9 277-82.
6 Miller C. Ensuring continuing care: styles and efficiency of the handover process. Nurs Older People 2006; 18: 32-6.

Ömür Budanur Miles ST3 trainee, Central Bristol Learning DisabilityTeam, Bristol,email: omurmiles@doctors.org.uk

\section{SHARIF GHALI}

\section{On-site testing for drugs of misuse in the acute psychiatric ward}

\section{AIMS AND METHOD}

To explore why and how on-site urine drug testing is performed in in-patient settings. Data were collected by questionnaire in four acute psychiatric wards.

\section{RESULTS}

The most commonly cited reasons for testing were suspected drug use and

\author{
as a routine part of the admission \\ procedure. On-site testing was \\ typically favoured over laboratory \\ methods owing to the rapid \\ turnaround of results and ease of \\ use. $\ln 81 \%$ of cases the result of the \\ tests had no effect on immediate \\ management. The majority of staff \\ had not received formal training in \\ their use.
}

\begin{abstract}
CLINICAL IMPLICATIONS
Clinical use of on-site drug tests does not reflect their established limitations. Guidance is required to direct staff in the use of this commonly used assessment tool.
\end{abstract}

There is a growing body of evidence describing the hazards of substance misuse in those with mental illness. These include elevated risk of relapse of psychosis, ${ }^{1}$ increased frequency of psychiatric admission, ${ }^{2}$ increased suicidal behaviour, ${ }^{3}$ worse treatment outcomes, ${ }^{2}$ and elevated potential for antisocial behaviour and contact with the criminal justice system. ${ }^{4,5}$ Despite these findings, illicit drugs are readily available on acute psychiatric wards. In a sample of people with psychotic illness admitted to three inner-London psychiatric units, half showed evidence of substance or alcohol misuse, a quarter reported using cannabis on the ward in the past 6 months, and 1 patient in 25 described their first-ever experience of drug use occurring as an in-patient. ${ }^{6}$

Accordingly, substance misuse has required increasingly careful consideration in the assessment and management of the acute psychiatric in-patient, and testing for drugs of misuse is a tool frequently used by mental health professionals. The desire for rapid turnaround of results has resulted in the development of devices over the past two decades to assess drug use on-site, currently in the form of numerous commercial dipstick and cartridge tests. Despite the obvious advantages of such products in busy acute in-patient settings, concerns have been flagged up regarding their validity and reliability. ${ }^{7-11}$

This study describes the use of on-site testing for drugs of misuse in acute adult in-patient settings in one London specialist mental health trust.

\section{Method}

Data were collected over a 2-month period in 2007 by means of a specifically designed questionnaire. These were distributed to four acute psychiatric wards in Oxleas National Health Service (NHS) Foundation Trust, which spans three London boroughs. For each on-site test performed, the tester was required to provide the following information:

- the reason for testing for drugs of misuse;

- why on-site testing had been selected over laboratory testing;

- the results of the test;

- how the specimen was collected;

- the impact of the test result on immediate clinical management;

- the profession of the tester, their training in the use of on-site devices, and whether they were confident in interpreting the results.

For most fields, the tester was requested to select one of multiple options. Three fields allowed staff to select as many as applied, with the option of selecting 'other' and entering their own explanation. In order to maximise data collection, a f200 contribution to ward funds was made available and distributed according to the ward's level of participation in the study.

\section{Results}

Sixty-seven completed questionnaires were returned. On only $4 \%(n=3)$ of occasions was a single-drug (cannabis only) test used. In $91 \%(n=61)$ of cases, staff chose to use a multidrug device. In $4 \%$ of cases $(n=3)$ the test used was not specified. Testing was most commonly performed because of suspected drug use and as a routine part of the admission procedure. These were 